\title{
Diversidad genética y estructura poblacional del cangrejo del manglar (Ucides occidentalis) en Tumbes, Perú
}

\author{
Ordinola, Z.A.,; Vieyra, E.G. ; Ramírez, B.E. ${ }^{\text {; }}$; Saavedra, K.Y. ${ }^{2}$ \\ Facult. Ingen. Pesq. Univ. Nacional de Tumbes, Puerto Pizarro, Tumbes, Perú. \\ ${ }^{2}$ Escuela Posgrado Univ. Nac. de Tumbes, Av.Universitaria s/n, Tumbes, Perú. \\ E-mail: aordinolaz@untumbes.edu.pe
}

\begin{abstract}
Resumen
Ordinola, Z.A.; Vieyra, E.G.; Ramírez, B.E.; Saavedra, K.Y.: Diversidad genética y estructura poblacional del cangrejo del manglar (Ucides occidentalis) en Tumbes, Perú. Rev. Vet. 31: 1, 33-37, 2020. El manglar es uno de los ecosistemas costeros más deteriorado a nivel mundial. En el presente caso, su funcionamiento ecológico depende en parte de la actividad de la especie clave: Ucides occidentalis. Este cangrejo ha sido sobreexplotado en los manglares del Perú. La fuerte reducción de su población (hasta 35,8\% en 11 años) puede hacer necesario cultivar larvas de este cangrejo para repoblar los manglares. Sin embargo un adecuado repoblamiento requiere conocer su diversidad genética y estructura genética poblacional. Es por ello que en esta investigación se propuso determinar estos aspectos de U. occidentalis en el manglar de Tumbes, Perú. Un total de 56 ejemplares de U. occidentalis fueron colectados, de los cuales se obtuvieron 42 secuencias nucleótidas de un fragmento del gen COI. Los resultados indicaron un alto nivel de diversidad genética (evaluada a través del número de haplotipos: 30 , frecuencia del haplotipo más frecuente: $14,29 \%$, diversidad de haplotipos: 0,9721; promedio de diferencias en nucleótidos: 4,396 y diversidad nucleótida: 0,00810 ), así como una baja estructura genética poblacional evaluada mediante AMOVA (variabilidad genética entre poblaciones: $4 \%$ ).
\end{abstract}

Palabras clave: cangrejo, variabilidad genética, manglar, gen COI, biodiversidad.

\begin{abstract}
Ordinola, Z.A.; Vieyra, E.G.; Ramírez, B.E.; Saavedra, K.Y.: Genetic diversity and population structure of the mangrove crab (Ucides occidentalis) in Tumbes, Peru. Rev. Vet. 31: 1, 33-37, 2020. Mangrove is one of the most threatened coastal ecosystems on earth. Its ecological functioning depends in part of activity of key species Ucides occidentalis. The crab stocks have been overfished in mangroves of Peru. The strong decline of the crab population (up $35.8 \%$ in 11 years) may make it necessary to cultivate larvae of this crab to repopulate the mangroves. However, an adequate repopulation requires to know its genetic diversity and genetic population structure. Therefore, the present study aimed to determine these aspects of $U$. occidentalis in the mangroves of Tumbes, Peru. A total of 56 individuals of $U$. occidentalis were collected, of which we obtained 42 nucleotide sequences of the gene fragment COI. The results indicated a high level of genetic diversity (as assessed by the haplotypes number: 30 , the most frequency of more frequent haplotype: $14.29 \%$ and haplotype diversity: 0.9721 ; nucleotide differences average: 4.396 and nucleotide diversity: 0.00810 ), and a low population genetic structure evaluated by AMOVA (genetic variability between populations: $4 \%$ ).
\end{abstract}

Key words: crab, genetic variability, mangrove, COI gene, biodiversity.

\section{INTRODUCCIÓN}

El manglar es uno de los ecosistemas costeros que brinda más servicios ambientales y a la vez uno de los más amenazados a nivel mundial ${ }^{19}$. Su correcto funcionamiento depende de la actividad de varias especies que lo habitan. En el caso de los manglares en los que la vegetación predominante es el mangle rojo (Rhizophora mangle) -como es el caso de los manglares del

Recibido: mayo 2019 / Aceptado: agosto 2019 pacífico americano- la especie clave es el cangrejo del manglar Ucides occidentalis, que se encarga de reciclar entre el 75 y $84 \%$ de la materia orgánica de dicho ecosistema $^{1,20}$.

En el Perú, el ecosistema del manglar se ubica casi exclusivamente en la región Tumbes, abarcando 4541 ha y estando dominado por el mangle rojo ${ }^{13}$, con sólo 300 ha en la región Piura, que no cuenta con mangle rojo $^{7}$. La población de $U$. occidentalis se localiza mayormente en la zona Tumbes ${ }^{13}$. 
$U$. occidentalis es un cangrejo que habita las costas del Pacífico americano, desde la isla Espiritu Santo (México) hasta San Pedro de Vice en Piura (Perú) ${ }^{1}$, viviendo en la zona intermareal donde se alimenta de hojas de mangle rojo ${ }^{20}$. Es también el cangrejo más explotado en el manglar peruano, lo que ha originado que su población se reduzca un 35,8\% en el lapso de 11 años (Prospección bioecológica de Ucides occidentalis en Región Tumbes, 2010, 1ra edición, Ed. Instit Mar Perú, Tumbes, 20 p.).

Su situación es similar a la de $U$. cordatus en Brasil, el cual también redujo dramáticamente su población ${ }^{5,17}$ por lo cual a partir de 2001 se inició su repoblamiento liberando larvas cultivadas al medio natu$\mathrm{ral}^{6,9}$. Sin embargo, este esfuerzo se realizó de manera inadecuada, sin haber estudiado

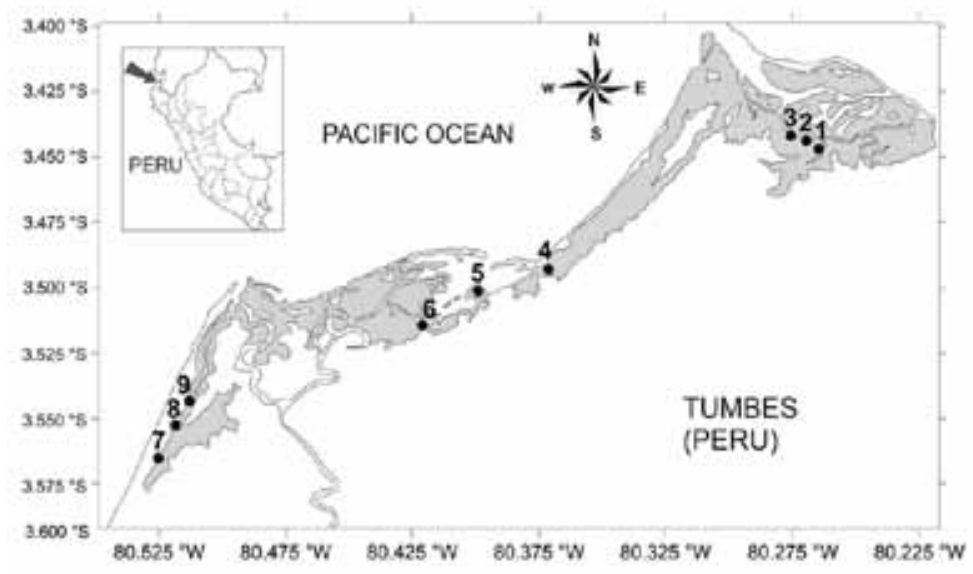

Figura 1. Sitios de muestreo de Ucides occidentalis en el manglar de la región Tumbes (Perú). 1: Zarumilla, 2: El Algarrobo, 3: Isla Matapalo, 4: El Jelí, 5: Isla El Tanque, 6: El Monteo, 7: La Chepa, 8: Corrales I y 9: Corrales II.

la diversidad genética o la estructura genética poblacional de este cangrejo, por lo que posteriormente tuvo que hacerse para mejorar las estrategias de redoblamiento ${ }^{16}$.

Es posible que a corto plazo se requiera realizar también repoblamiento de $U$. occidentalis en el manglar peruano, por lo que es necesario conocer la diversidad genética y la estructura genética poblacional del mismo, para poder orientar la estrategia de repoblamiento a realizar, que no solo debe incrementar el tamaño poblacional sino mantener la diversidad genética y evitar inducir niveles altos de estructuración genética poblacional ${ }^{12}$.

La evaluación de la diversidad genética y de la estructura genética poblacional, se puede realizar secuenciando un fragmento del gen de la subunidad I de la citrocromo oxidasa (COI), tal como se ha empleado reiteradas veces en estudios similares en plantas y animales, incluyendo los crustáceos ${ }^{2,10,11,23}$.

Esta investigación tuvo como objetivo determinar la diversidad genética y la estructura genética poblacional de $U$. occidentalis en el manglar de la región Tumbes (Perú).

\section{MATERIAL Y MÉTODOS}

Recolección de la muestra. Una muestra de 55 ejemplares de $U$. occidentalis se recogió de nueve estaciones de muestreo en el manglar de Tumbes (Perú) (Figura 1). Los cangrejos fueron llevados para su análisis al Laboratorio de Biología Molecular de la Universidad Nacional de Tumbes (Puerto Pizarro, Perú).

Obtención de muestras de músculo. Se extrajo $0,1 \mathrm{~g}$ de músculo de $U$. occidentalis y se colocó en un microtubo de centrífuga de $1,5 \mathrm{ml}$, añadiéndole $1 \mathrm{ml}$ de etanol al $95 \%$.

Extracción de ADN. Se realizó utilizando el protocolo de extracción con bromuro de cetil trimetil amonio (CTAB), tal como lo efectuaron acertadamente otros investigadores en $2017^{15}$.
Amplificación del fragmento del gen COI (por reacción en cadena de la polimerasa, PCR). La amplificación se llevó a cabo en un volumen de reacción de $25 \mu 1$, conteniendo $16,75 \mu 1$ de agua ultra pura, $2,50 \mu 1$ de buffer 10X PCR RXn (marca Invitrogen), 2,50 $\mu \mathrm{l}$ de $\mathrm{MgCl}_{2} 10 \mathrm{mM}$ (marca Invitrogen), 0,50 $\mu 1$ de mix $10 \mathrm{mM}$ de desoxinucleótidos trifosfato (dNTPs, marca Fermentas), 1,00 $\mu 1$ de albúmina de suero bovino (BSA), $0,50 \mu 1$ de iniciador forward LCO1490 y 0,50 $\mu \mathrm{l}$ de iniciador reverse HCO2198 (ambos a $10 \mathrm{pMol} /$ $\mu 1$, marca Fermentas), 0,25 $\mu 1$ de Taq polimerasa (5 U/ $\mu 1$, marca Invitrogen) y $0,50 \mu \mathrm{l}$ de la muestra de ADN. La amplificación se realizó en un termociclador (marca Techne modelo FTC3102D) con pre desnaturalización a $95^{\circ} \mathrm{C}$ por $1 \mathrm{~min}$, seguido de 40 ciclos de amplificación (desnaturalización a $94^{\circ} \mathrm{C}$ por $30 \mathrm{~s}$, hibridación a $50^{\circ} \mathrm{C}$ por $45 \mathrm{~s}$ y polimerización a $72^{\circ} \mathrm{C}$ por $1 \mathrm{~min}$ ) seguido de una etapa de polimerización final a $72^{\circ} \mathrm{C}$ por $7 \mathrm{~min}$.

Secuenciación del ADN. Cuarenta y cinco (45) muestras de ADN amplificadas exitosamente fueron enviadas para su secuenciamiento a Macrogen (Maryland, USA), obteniéndose 42 secuencias.

Análisis de las secuencias nucleotídicas. Las 42 secuencias nucleotídicas fueron alineadas en el software Mega versión 5, y comparadas con dos secuencias del fragmento del gen COI de $U$. occidentalis obtenidas de Genbank (números de acceso: JX524479 y JX524480) para descartar nucleótidos no confiables en las secuencias investigadas, después de lo cual la longitud de las secuencias se redujo a $543 \mathrm{pb}$. Las secuencias depuradas fueron depositadas en GenBank, (números de acceso: JX564556 - JX564597).

Análisis de la diversidad genética y la estructura genética poblacional. Las secuencias fueron analizadas con los software Mega 5 y DnaSP 5, para determinar la composición nucleotídica, el número de haplotipos (Nh), la diversidad de haplotipos (h) y la diversidad 
nucléotida $(\pi)$. Con GenAlex 6 se obtuvo la matriz de distancia genética de $\mathrm{Nei}$, se evaluó el nivel de estructura genética poblacional usando análisis molecular de varianza (AMOVA) y la correlación entre distancias genéticas y geográficas mediante el test de Mantel.

\section{RESULTADOS}

Diversidad genética poblacional. La diversidad genética de $U$. occidentalis fue alta (Tabla 1), según lo indica su número de haplotipos: 30 en 42 individuos. Tres haplotipos (Hap2, Hap3 y Hap5) estuvieron presentes simultáneamente en las tres zonas de estudio (Zarumilla, Tumbes y Corrales), dos lo estuvieron en dos zonas (Hap6 en Zarumilla y Corrales, y Hap9 en Puerto Pizarro y Corrales).

Los 25 haplotipos restantes se hallaron distribuidos en una sola zona. La frecuencia de haplotipos varió entre $2,38 \%$ para los menos frecuentes, y $14,29 \%$ para el más frecuente (Hap3) hallado en seis individuos de los 42 muestreados. El promedio de diferencias nucleótidas fue de 4,396 nucleótidos en la secuencia de $543 \mathrm{pb}$ y la diversidad nucléotida $(\pi)$ fue de 0,00810 .

La distancia genética fue baja y varió entre 0,001 a 0,005 (descartando las distancias dentro de la misma estación de muestreo). La mayor distancia genética se dio entre la estación de muestreo de El Jelí y la estación del estero Corrales (Tabla 2).

Estructura genética poblacional. El AMOVA mostró que la variabilidad genética se debió en un 96\% a las variaciones genéticas de los individuos dentro de las poblaciones y en un $4 \%$ a la variación entre poblaciones. El test de Mantel presentó un coeficiente de correlación de Pearson casi igual a cero $(r=-0,032)$, indicando la inexistencia de una correlación entre la distancia geográfica y la distancia genética evaluada.

\section{DISCUSIÓN}

La diversidad genética de $U$. occidentalis fue alta, lo que se expresa a través de un elevado número de haplotipos (30 de 42) y la existencia de polimorfismo, pues
Tabla 1. Frecuencia absoluta $\left(\mathrm{N}^{\circ}\right)$ y porcentual (\%) de haplotipos del gen COI en Ucides occidentalis según zona de muestreo en los manglares de Tumbes, Perú.

\begin{tabular}{|c|c|c|c|c|c|c|c|c|}
\hline & \multicolumn{2}{|c|}{$\begin{array}{l}\text { zona norte } \\
\text { Zarumilla }\end{array}$} & \multicolumn{2}{|c|}{$\begin{array}{c}\text { zona centro } \\
\text { Puerto Pizarro }\end{array}$} & \multicolumn{2}{|c|}{$\begin{array}{l}\text { zona sur } \\
\text { Corrales }\end{array}$} & \multicolumn{2}{|c|}{ total } \\
\hline & $\mathrm{N}$ & $\%$ & $\mathrm{~N}^{\circ}$ & $\%$ & $\mathrm{~N}^{\circ}$ & $\%$ & $\mathrm{~N}^{\circ}$ & $\%$ \\
\hline Hap1 & 0 & 0,00 & 0 & 0,00 & 1 & 7,69 & 1 & 2,38 \\
\hline Hap2 & 1 & 5,88 & 1 & 8,33 & 1 & 7,69 & 3 & 7,14 \\
\hline Hap3 & 1 & 5,88 & 2 & 16,67 & 3 & 23,08 & 6 & 14,29 \\
\hline Hap4 & 0 & 0,00 & 0 & 0,00 & 1 & 7,69 & 1 & 2,38 \\
\hline Hap5 & 1 & 5,88 & 1 & 8,33 & 1 & 7,69 & 3 & 7,14 \\
\hline Hap6 & 1 & 5,88 & 0 & 0,00 & 1 & 7,69 & 2 & 4,76 \\
\hline Hap7 & 0 & 0,00 & 0 & 0,00 & 1 & 7,69 & 1 & 2,38 \\
\hline Hap8 & 0 & 0,00 & 0 & 0,00 & 2 & 15,38 & 2 & 4,76 \\
\hline Hap9 & 0 & 0,00 & 1 & 8,33 & 1 & 7,69 & 2 & 4,76 \\
\hline Hap10 & 0 & 0,00 & 0 & 0,00 & 1 & 7,69 & 1 & 2,38 \\
\hline Hap11 & 0 & 0,00 & 1 & 8,33 & 0 & 0,00 & 1 & 2,38 \\
\hline Hap12 & 0 & 0,00 & 1 & 8,33 & 0 & 0,00 & 1 & 2,38 \\
\hline Hap13 & 0 & 0,00 & 1 & 8,33 & 0 & 0,00 & 1 & 2,38 \\
\hline Hap14 & 0 & 0,00 & 1 & 8,33 & 0 & 0,00 & 1 & 2,38 \\
\hline Hap15 & 0 & 0,00 & 1 & 8,33 & 0 & 0,00 & 1 & 2,38 \\
\hline Hap16 & 0 & 0,00 & 1 & 8,33 & 0 & 0,00 & 1 & 2,38 \\
\hline Hap17 & 0 & 0,00 & 1 & 8,33 & 0 & 0,00 & 1 & 2,38 \\
\hline Hap18 & 1 & 5,88 & 0 & 0,00 & 0 & 0,00 & 1 & 2,38 \\
\hline Hap19 & 1 & 5,88 & 0 & 0,00 & 0 & 0,00 & 1 & 2,38 \\
\hline Hap20 & 1 & 5,88 & 0 & 0,00 & 0 & 0,00 & 1 & 2,38 \\
\hline Hap21 & 1 & 5,88 & 0 & 0,00 & 0 & 0,00 & 1 & 2,38 \\
\hline Hap22 & 1 & 5,88 & 0 & 0,00 & 0 & 0,00 & 1 & 2,38 \\
\hline Hap23 & 1 & 5,88 & 0 & 0,00 & 0 & 0,00 & 1 & 2,38 \\
\hline Hap24 & 1 & 5,88 & 0 & 0,00 & 0 & 0,00 & 1 & 2,38 \\
\hline Hap25 & 1 & 5,88 & 0 & 0,00 & 0 & 0,00 & 1 & 2,38 \\
\hline Hap26 & 1 & 5,88 & 0 & 0,00 & 0 & 0,00 & 1 & 2,38 \\
\hline Hap27 & 1 & 5,88 & 0 & 0,00 & 0 & 0,00 & 1 & 2,38 \\
\hline Hap28 & 1 & 5,88 & 0 & 0,00 & 0 & 0,00 & 1 & 2,38 \\
\hline Hap29 & 1 & 5,88 & 0 & 0,00 & 0 & 0,00 & 1 & 2,38 \\
\hline Hap30 & 1 & 5,88 & 0 & 0,00 & 0 & 0,00 & 1 & 2,38 \\
\hline total & 17 & 100 & 12 & 100 & 13 & 100 & 42 & 100 \\
\hline
\end{tabular}

Tabla 2. Matriz de distancia genética de Nei según zona y estación de muestreo en Ucides occidentalis en el manglar de Tumbes, Perú.

\begin{tabular}{|c|c|c|c|c|c|c|c|c|c|}
\hline \multicolumn{3}{|c|}{ zona norte (Zarumilla) } & \multicolumn{3}{|c|}{ zona centro (Pizarro) } & \multicolumn{3}{|c|}{ zona sur (Corrales) } & \multirow{2}{*}{$\begin{array}{l}\text { estación } \\
\text { de } \\
\text { muestreo }\end{array}$} \\
\hline $\begin{array}{l}\text { Alga- } \\
\text { rrobo }\end{array}$ & Mata-palo & Zaru-milla & $\begin{array}{c}\text { El } \\
\text { Jelí }\end{array}$ & $\begin{array}{c}\text { Mon- } \\
\text { teo }\end{array}$ & $\begin{array}{l}\text { I.Tan- } \\
\text { que }\end{array}$ & $\begin{array}{l}\text { Cor- } \\
\text { ral. I }\end{array}$ & $\begin{array}{l}\text { Cor- } \\
\text { ral.II }\end{array}$ & $\begin{array}{c}\text { Che- } \\
\text { pa }\end{array}$ & \\
\hline 0,000 & & & & & & & & & El Algarrobo \\
\hline 0,001 & 0,000 & & & & & & & & Isla Matapalo \\
\hline 0,002 & 0,002 & 0,000 & & & & & & & Zarumilla \\
\hline 0,002 & 0,001 & 0,002 & 0,000 & & & & & & El Jelí \\
\hline 0,003 & 0,001 & 0,002 & 0,003 & 0,000 & & & & & El Monteo \\
\hline 0,003 & 0,001 & 0,001 & 0,002 & 0,002 & 0,000 & & & & Isla El Tanque \\
\hline 0,002 & 0,001 & 0,001 & 0,005 & 0,001 & 0,001 & 0,000 & & & Corrales I \\
\hline 0,001 & 0,001 & 0,002 & 0,001 & 0,001 & 0,002 & 0,002 & 0,000 & & Corrales II \\
\hline 0,002 & 0,001 & 0,002 & 0,002 & 0,001 & 0,002 & 0,003 & 0,001 & 0,000 & La Chepa \\
\hline
\end{tabular}


el haplotipo más frecuente (Hap3) tuvo una frecuencia menor al $95 \%{ }^{12}$. El polimorfismo es frecuente en el gen COI: se ha reportado en varios crustáceos, entre ellos los cangrejos Eriocheir sinensis ${ }^{23}$, Procambarus clarkii $^{2}$ y Scylla paramamosain ${ }^{14}$. También ha sido reportado en $U$. cordatus ${ }^{10}$ en el cual el haplotipo más frecuente tuvo una frecuencia de $15,7 \%$, que es bastante similar a la hallada en esta investigación. La razón de este polimorfismo es que al ser un gen mitocondrial tiene alta tasa de mutación ${ }^{3}$ con lo que es probable un alto número de haplotipos.

$U$. occidentalis tuvo una diversidad de haplotipos: $\mathrm{Hd}=0,9721$, lo que también indica una alta diversidad. En varios crustáceos se ha observado un alto índice de diversidad de haplotipos cuando se evalúa el gen COI. Así, en el cangrejo del fango (Scilla paramamosain) se ha reportado Hd entre 0,625 y $0,914{ }^{14}$; en el camarón de río (Macrobrachium amazonicum) de $0,8488^{22}$ y en U. cordatus de $0,97^{10}$, siendo esta última muy similar a la encontrada para $U$. occidentalis tal vez por tratarse de especies del mismo género.

La diversidad nucléotida ( $\pi$ ) para el gen COI de $U$. occidentalis fue de $0,00810(0,8 \%)$; otros investigadores también han hallado valores similares en otros crustáceos, como en los cangrejos Austropotamobius pallipes y A. torrentium con valores de 0,001 a $0,034^{21}$, en el cangrejo Scylla paramamosain con 0,001 a $0,003{ }^{14}$, en el camarón Macrobrachium amazonicum con 0,0005 a $0,02422^{22}$, así como en $U$. cordatus con 0,0063 a $0,0065^{10}$. Esto muestra que la alta diversidad nucleótida de $U$. occidentalis es una característica compartida con otros crustáceos.

La estructura genética poblacional de U. occidentalis fue muy baja, del $100 \%$ de la variabilidad genética, $96 \%$ se da intra poblaciones y sólo $4 \%$ inter poblaciones, lo que indica que las poblaciones en las zonas estudiadas son bastante similares. Este resultado es semejante al hallado en $U$. cordatus al usar microsatélites ${ }^{4}(92,2 \%$ de variabilidad intra poblacional y sólo $7,8 \%$ inter poblacional), así como usando el gen COI ${ }^{10}$ ( $97,44 \%$ de variabilidad intra poblacional y $2,56 \%$ inter poblacional).

El análisis de Mantel realizado a $U$. occidentalis mostró que la distancia genética no tiene correlación con la distancia geográfica. Estudios con U. cordatus ${ }^{4,8,10}$ han concluido que en dicha especie tampoco existe correlación, incluso poblaciones de $U$. cordatus distantes más de $4500 \mathrm{~km}$ y separadas por la desembocadura del río Amazonas (que podría suponer una barrera geográfica importante) se mostraron genéticamente similares ${ }^{16}$.

La baja estructuración genética poblacional observada en $U$. occidentalis se justifica por que al igual que $U$. cordatus tiene un alto flujo génico que se desarrolla por la eficiente dispersión larval, pues los estadios posteriores a Zoea II salen a mar abierto ${ }^{10}$ permaneciendo allí por 20 a 69 días, tiempo en que las larvas pueden ser trasportadas por las corrientes varios miles de kilómetros, permitiendo el intercambio efectivo de genes ${ }^{4,16}$.
La alta diversidad genética y la baja estructuración genética poblacional de $U$. occidentalis en los manglares de Tumbes (Perú), indican que el repoblamiento del mismo en el manglar tumbesino es posible utilizando un stock de reproductores no muy elevado al contarse con una población con alta diversidad genética ${ }^{18}$.

El esfuerzo de recolectar el stock de reproductores depende de la estructura genética poblacional: si es alta, la selección de reproductores se dará en cada una de las subpoblaciones para conservar los alelos exclusivos de cada una de ellas; si es baja la selección puede realizarse considerándose una población única ${ }^{18}$.

Por ello, un futuro repoblamiento de $U$. occidentalis en el manglar de Tumbes, se podría realizar utilizando una cantidad baja de reproductores recolectados en pocas zonas del manglar.

Agradecimiento. A la Ing. Pesq. Carolina Solano Chávez, por su apoyo en la amplificación del ADN de algunas muestras.

\section{REFERENCIAS}

1. Alemán S et al. 2017. Ampliación de la distribución sur de Ucides occidentalis (Decapoda: Ucididae) y Cardisoma crassum (Decapoda: Gecarcinidae). Rev Peruana Biol 24: 107-110.

2. Almerão MP et al. 2018. Genetic diversity of the invasive crayfish Procambarus clarkii in France. Limnologica 69: 135-141.

3. Arif IA, Khan HA. 2009. Molecular markers for biodiversity analysis of wildlife animals: a brief review. Anim Biodivers Conserv 32: 9-17.

4. Britto FB et al. 2011. Research note single primer-based DNA amplification as a suitable and low-cost tool for assessing genetic diversity in mangrove crabs. Genet \& Molec Res 10: 4084-4092.

5. Britto FB et al. 2018. Population connectivity and larval dispersal of the exploited mangrove crab Ucides cordatus along the Brazilian coast. https://peerj.com/articles /4702/

6. De Britto SD, Abrunhosa FA, Diele K. 2017. Metamorphosis of the edible mangrove crab Ucides cordatus (Ucididae) in response to benthic microbial biofilms. $J$ Exp Marine Biol \& Ecol 492: 132-140.

7. Charcape RM, Moutarde F. 2005. Diversidad florística y conservación del Santuario Regional de Piura Manglares San Pedro de Vice-Sechura. Rev Peruana Biol 12: 327-334.

8. Cottens KF. 2009. Efeitos da temperatura, intensidade luminosa e da densidade de cultivo na larvicultura de $U c i$ des cordatus (Linnaeus, 1763) (Crustacea, Decapoda. Brachyura) em laboratorio. Dissertação de Mestrado, Universidade Federal do Paraná, Curitiba, Brasil, 82 p.

9. Cottens KF et al. 2014. Cultivo de larvas de Ucides cordatus (Linneau, 1763) sob diferentes intensidades luminosas. Arq Bras Med Vet Zoot 66: 1464-1470.

10. Ewald M. 2006. Isolation and characterization of nuclear and mitochondrial genetic markers for population studies of Ucides cordatus cordatus (Decapoda: Brachyura). Doctor dissertation, University of Bremen, Bremen, $101 \mathrm{p}$. 
11. Ferrer MS et al. 2018. Genetic diversity of Philippine Gracilaria salicornia (Gracilariaceae, Rhodophyta) based on mitochondrial COI-5P sequences. Biologia 12: 1-9.

12. González EA. 2011.Análisis de la diversidad genética en poblaciones naturales de especies vegetales amenazadas: Ilex perado sp lopezlilloi (Aquifoliaceae), Silene nocteolens (Caryophyllaceae) y Sorbus aria (Rosaceae). Tesis Doctoral, Uni-versidad de las Palmas de Gran Canaria, Las Palmas de Gran Canaria, 238 p.

13. INRENA. 2007. Plan Maestro del Santuario Nacional Los Manglares de Tumbes 2007-2011, 1ra edición, Inrena, Lima, Perú, 197 p.

14. Ma H, Ma C, Ma L. 2011. Population genetic diversity of mud crab (Scylla pa-ramamosain) in Hainan Island of China based on mitochondrial DNA. Biochem Systemat \& Ecol 39: 434-440.

15. Mendoza O et al. 2017. Respuesta fisiológica y molecular de Anadara tuberculosa (Arcoida: Arcidae) al estrés de salinidad. Rev Biol Trop 65: 1142-1151.

16. Oliveira JF. 2009. Filogeografia e demografia evolutiva de Ucides cordatus (Linnaeus 1763) e Cardisoma guanhumi (Latreille, 1825) na costa do Brasil. Tese de Doutor, Universidade Federal do Paraná, Curitiba, Brasil. 118 p.

17. Orélis RR, Chammas MA, Ostrensky A, Boeger WA. 2012. Viability of the etiologic agent of the Lethargic Crab Disease, Exophiala cancerae, during cooking of the mangrove-land crab. Food Control 25: 591-593.
18. Page KS, Scribner KT. 2004. Genetic diversity of wild and hatchery lake trout populations: relevance for management and restoration in the Great Lakes. Trans Am Fish Soc 133: 674-691.

19. Sarker SK et al. 2016. Are we failing to protect threatened mangroves in the Sundarbans world heritage ecosystem? Scientific Reports 6: 21234.

20. Solano F. 2006. Aspectos pesqueros biológicos y socioeconómicos de la captura de cangrejo rojo (Ucides occidentalis) en los manglares del Ecuador, 1ra edición, Instituto Nacional de Pesca, Guayaquil, 32 p.

21. Trontelj P, Machino Y, Sket B. 2005. Phylogenetic and phylogeographic relation-ships in the crayfish genus Austropotamobius inferred from mitochondrial COI gene sequences. Molec Phylogen \& Evolut 34: 212-226.

22. Vergamini FG, Pileggi LG, Mantelatto FL. 2011. Genetic variability of the Amazon River prawn Macrobrachium amazonicum (Decapoda, Caridea, Palae-monidae). Contrib Zool. 80: 67-83.

23. Zhang C, Li Q, Wu X, Liu Q, Cheng Y. 2018. Genetic diversity and genetic structure of farmed and wild Chinese mitten crab (Eriocheir sinensis) populations from three major basins by mitochondrial DNA COI and Cyt b gene sequences. Mi-tochondrial DNA Part A 29: 1081-1089.

\section{Revista Veterinaria ingresa a SciELO}

Revista Veterinaria, publicación oficial de la Facultad de Ciencias Veterinarias de la Universidad Nacional del Nordeste (Corrientes, Argentina), ha logrado acceder al Núcleo Básico de Revistas Científicas Argentinas (Nivel 1), luego de calificar adecuadamente en el Centro Argentino de Información Científica y Tecnológica (CAICYT), según Resolución 2485/14 del Consejo Nacional de Investigaciones Científicas y Técnicas (CONICET).

Sobre un puntaje máximo de 33 se obtuvieron 32 puntos. Tal calificación constituye "una garantía de la excelencia de la publicación" (sic) y queda expedita la vía del Portal SciELO (Scientific Electronic Library Online) para los artículos publicados.

En tal calificación gravitó positivamente la circunstancia de haber aumentado el índice de impacto (Scimago-Elsevier) y haber disminuido las autocitaciones. También se tuvieron en cuenta aspectos como la amplia cobertura de la revista, la calidad científica del Comité Editorial, los criterios de evaluación de los artículos, el origen de los autores (locales 60\%, nacionales 13\%, extranjeros $24 \%$, en idioma inglés), el adecuado balance entre trabajos científicos originales y reseñas bibliográficas (ambos con alta calidad), así como el estricto cumplimiento de la periodicidad semestral y la favorable acogida por indizadores como Cab, J-Gate, Doaj, Ebsco, Gale Cengage, Infocyt, Latindex y Scopus.

Se consolida de esta manera la continuidad de "Revista Veterinaria", que en su acontecer registra más de 50 años de existencia en nuestra Facultad de Ciencias Veterinarias, entidad que en 2019 cumplió el 99 aniversario de su fundación. 